\section{Virtual Reality Interface for Accessing Electronic Information}

\author{
Felix Moya Anegon, Victor \\ Herrero-Solana, Vicente \\ Guerrero-Bote
}

\begin{abstract}
The Authors
FĖlix de Moya-AnegÛn is Professor of Advanced Information Retrieval Systems and Dean of the Library and Information Science Faculty at the University of Granada, Spain.
\end{abstract}

Vİctor Herrero-Solana is Professor of Computer Science in the Library and Information Science Department. University of Mar del Plata, Argentina.

Vicente Guerrero-Bote is Professor of Computer Science in the Library and Information Science Faculty, University of Extremadura, Spain.

Those heading the project may be reached at this address:

FÈlix de Moya AnegÛn

Library and Information Studies Faculty

University of Granada

E-mail: HIPERVÕNCULO mail to:

felix@goliat.ugr.es

Phone: (34) 58243943

Fax: (34) 58243945

\section{Background}

Advanced techniques for Information Retrieval (IR) currently make up one of the most active areas for research in the field of library and information science. New models representing document content are replacing the classic systems in which the search terms supplied by the user were compared against the indexing terms existing in the inverted files of a database.

The purpose of such models is threefold: first, to facilitate the recovery of documents in order of relevance for the user; secondly, to create a documental representation that makes browsing easier; and third, to render unnecessary the boolean coordinates. Therefore, the generalised use of these interface models will allow end users simplified and homologous access to a given body of documents on the basis of the knowledge contained therein.

Today's alternative models are based on the mathematic concept known as "similarity coefficient" (Moya, F. 1995). One of the best known is the vector space model defined by Salton within his project SMART (Salton, G. 1979): documents are represented by $n$ dimensional vectors, $n$ being the number of indexing terms contained in the complete text. This multidimensional space faithfully represents the complexity of content relationships within a specific document collection. Nonetheless, as it is impossible to visualise this space, the user must resort to using a series of algorithms denominated "dimension reduction algorithms," which make it possible to generate an observable approximate representation of the material in question.

The most prevalent algorithms are:

1) Principal component analysis (PCA),

2) Multidimensional scaling (MDS) and

3) Cluster analysis (Kinnucan, M., Nelson, M. and Allen, B. 1987). 
A fourth possibility would be to process information by means of artificial neural networks (ANNs), specifically, the ANN model called the Self-Organizing Map (also known as the SOM or Kohonen model). It is based on the principle of self organization and grouping of $n$-dimensional vectors in a bidimensional space (Kohonen, T. 1997). This model has been used to reduce dimensions in a wide variety of document spaces of diverse nature (Honkela, T.et.al. 1995, 1996a, 1996b) (Kaski, S. et.al. 1995, 1996) (Lagus, K. et al . 1996) (Lin, X. 1995, 1996, 1997).

The output obtained using any one of the algorithms consists of a series of coordinates in a reduced space (usually bidimensional), thereby allowing a plain visualization, albeit with some stress, of the relationships existing in the multidimensional space.

Three dimensional models Bearing in mind that, to date, no more than three dimensions can be clearly represented in graphic space, the proposed research project introduces the premise that a 3D space can be used as reduced space in order to limit the stress of the representation, and produce a model more closely resembling multidimensional reality. This advancement would mean an accurate, realistic representation of the complex content relationships among documents, and would thus simplify the user's search for and retrieval of the desired information.

The problem is that $3 \mathrm{D}$, while generating less stress, has its limitations as far as maneuverability is concerned (Boyle, J., Lowit, P. and Mitchell, K. 1996) - although the plug-in VRMLs currently in use on the Internet have improved to such an extent that they afford users with considerable freedom of movement in worlds of virtual reality. We believe that the key to its success can be found in the use of a properly designed visualization metaphor. This is an immensely difficult task, as a metaphor by definition should make interaction simple and totally transparent for the user by way of its analogy with a much more complex system (Eberts, R. 1994); yet, at the same time, a metaphor is necessarily an incomplete and biased representation of the system (Dieberger, A. 1997).

The main challenge faced, then, is this: the final product will necessarily be a compromise between concretion and abstraction. A highly abstract metaphor would represent the structural organization of the body of documents with greater freedom, but runs the risk of 'losing' the user if its abstractions are beyond his or her grasp. Overly concrete representations, on the other hand, which are better rooted in the real world and appeal more to the users' intuitive faculties, are not able to represent adequately the structure of the documental body as a whole. Moreover, using them can prove to be tedious in the long-run.

Metaphors In Information Science the development of metaphors has been closely associated with the history of user interfaces, and has proved to be a determining factor for the consolidation of certain systems. The best known case in point is the desk metaphor, invented by Xerox and made popular by Apple in their Macintosh line, then adopted by Microsoft for the Intel-PC after its victory in the standards war (Lynch, P. 1994). Current trends suggest the creation of interfaces that are increasingly more accessible and better integrated with respect to the user (Piscitelli, A. 1995). Virtual Reality (VR) and 3D representations play an important role in this sense, and will gain in importance once the VRML (Virtual Reality Model Language) standard is established.

In a VR environment, the visualization metaphor takes on a leading role, reinforced by the wide range of forms it may adopt: geometric forms (cubes, spheres, polyhedral, etc.), artificial forms (houses, rooms, cities), natural forms (terrain in relief, trees), systematic structures (highway systems, 
water flow systems), dynamic systems (atoms, molecules, constellations), and traditional symbolic systems (mandalas, etc.) (Benking, H. and Judge, A. 1994).

The 3D metaphors developed to date can be divided into two main categories: those based on spatial nodes, and those based on structured maps (Andrews 1994). The former are often used to navigate through hypertext environments (most often, the Web) via bunches of interconnected nodes; whereas the latter are more appropriate for representing highly complex structural relationships, such as the document content of a given database.

One good example of the first group is the Narcissus model, developed at the University of Birmingham, for the tridimensional organization of Web information (Hendley, R. et.al. 1995). This is done through a conglomeration of constellations and galaxies of spheres of differing size that are interconnected by lines. The spheres represent pages, and the lines represent links. Based on this model, the authors have developed a special application called Hyperspace, designed for the management of Internet information (Wood, A. et.al. 1995a, 1995b).

One example of the second category, the structural maps, can be found in a prototype named Lyberworld (Hemmje, M. et.al. 1994, 1995). This consists of a graphic user interface developed for a probabilistic system of full text retrieval called INQUERY (Hemmje, M . 1993). Lyberworld presents two metaphors: "relevance spheres," for information retrieval, and "navigation cones," for database browsing. The cones are relatively simple hierarchic trees in $3 \mathrm{D}$, whereas the relevance spheres are concerned with the spatial organization of a specific group of documents according to their degree of relevance. These spheres may be used to represent the relationships of content and grades of pertinence existing in any single set of documents responding to the user's search. The user's interaction with the sphere, then, extends to the point of allowing only the most pertinent documents to be selected.

There are other recent creations that do not fit into either of the two categories mentioned above. Such is the case of Virgilio, an interface that visualises searches for musical works in a database (Levialdi, S., Massari, A. and Saladini, L. 1995). The drawback with Virgilio is that the chosen metaphor is a building (artificial form) which attempts to recreate a photographic-type VR environment, but proves difficult to navigate because of the fact that its operation does not take the user's intuition into account.

\section{Research Objectives}

The prime objective of the proposed research project is the development of a metaphor that would allow users to navigate among documents represented in a reduced space using one of the algorithmic methods described earlier (PCA, MDS, clustering and $\mathrm{SOM})$. For this purpose, the different existing metaphors are studied in order to synthesise the most significant elements of each one; then, on the basis of this analysis, an improved model of our own can be devised. The new metaphor will be subjected to a series of tests so that we may analyse the way in which it organises different types of information:

- Data structured hierarchically by means of a classic classification system (UDC, DDC, etc.);

- Information, also with a hierarchic structure, existing in Web directories (Yahoo, Galaxy, etc.); and

- Documents represented by the vector model. 
Virtual Reality Tnterface for Accessing Electronic Information

Anegon, Herrero-Solana, Guerrero-Bote

The latter is the most important group, and is subdivided into two phases:

- The visualization of the results of a query, and

- Browsing over the complete database.

The analytical study would include collaboration with users to assess the overall yield of the system as well as the degree to which the user adapts to and accepts the new work environment. One aspect deserving special attention is the observation of users interacting simultaneously in the system. We believe that in any electronic information retrieval system, interaction and communication with other users is a crucial factor that has been studied very little up until now. This type of cooperative navigation and user intercommunication provides information whose value may exceed the content of the system itself.

The second main objective is to study in depth the capacity of the SOM (SelfOrganizing Map) for reducing dimensions. Although we generally speak of four algorithms, the SOM is of particular interest to us because of its radically different approach to the problem of reduction. It will be the subject of a comparative analysis with respect to the other three types of algorithms to determine how space is organised and to what degree stress is introduced into the representations. To date, no study of this sort has been undertaken in the field of IR.

Although the specific technological tools to be applied have not yet been selected, it is clear that standard data formats such as VRML will be included. This guarantees the possibility of developing easy-to-share and easily portable applications. The use of proprietary formats severely conditions overall yield, as migration to other systems is impeded.

It is important to note that our principal aim is to develop a standard work environment - i.e. one not designed for specific contexts but rather for generalised use by a wide range of users in a great diversity of situations. For this reason it is very important that we work with the classic visualizers (Netscape and Explorer) and not with the reduced coverage of proprietary programs. Nonetheless, because the classic visualizers are not yet capable of functioning in multi-user environments, one possibility for the early stages of the project would be to study user interaction in a proprietary environment such as Alphaworlds SDK.

Because we believe that a system of information retrieval must be greater than the sum of its parts, communication should never be reduced to a simple back-and-forth between system and user. Aside from the more familiar types in inter-user communication (mailings, chats, avatars, etc.), we intend to explore the potential of the users themselves as sources of information with a significant added value: the value derived from one's own experience using the system. The interchange of this particular sort of information reinforces the sense of 'bonding' between user and medium. In this way, the system eventually becomes the user's territory, so to speak.

\section{Projected research}

The proposed research project draws from a university setting, and entails the collaboration of professors and students of Library and Information Science, principally from the University of Granada. In addition, formalised agreements with two organisations specialising in electronic information management make possible the technological transfer of our research findings. These organisations will also contribute with the human resources needed for analysing real work situations. The results of evaluation can therefore be directed back to the development process, and in this way, the best possible adaptation to the users' needs can be achieved. Likewise, the link 
between the new development and commercial products for document management already in existence may be put to the test by one of the participating institutions to demonstrate its potential.

This project is to be carried out in the framework of the research group denominated "Virtual reality interface for accessing electronic information" with funding from Spain's National Plan for Research and Development $(\mathrm{I}+\mathrm{D})$ through the Inter ministerial Commission of Science and Technology (CICYT). This plan covers the finance of advanced research in diverse technological areas; the project described here corresponds to the telecommunications area of the National Plan, under code number TEL97-1131.

\section{References}

Benking, $\mathrm{H}$ and Judge, $\mathrm{A}$ (1994) Design considerations for spatial metaphors, ECHT94 Workshop on Spatial Metaphors.

http://www.lcc.gatech.edu/faculty/dieberger/ECHT94. WS.Benking.html

Boyle, J Lowit, P and Mitchell, K (1996) 3D or not 3D.... Proceedings of the 3rd. FADIVA Workshop. Gubbio (Italy).

ftp://ftp.dis.uniromal.it/pub/santucci/in/FADIVA/jboyle. ps.z

Dieberger, A (1997) Navigation metaphors and soçial navigation in information spaces, CHI¥97 Workshop on Navigation in Information Spaces.

http://www.lcc.gatech.edu/ dieberger/CHI97_navigatio n_workshop.html

Eberts, R (1994) User interface design, Englewood Cliffs, Prentice Hall, (Prentice Hall International Series in Industrial and Systems Engineering).

Hemmje, M (1993) A 3D based user interface for information retrieval systems, Proceedings of IEEE Visualization'93. San Jose, California, 25-29 October. ftp://ftp.darmstadt.gmd. de/pub/VISIT/papers/hemmje/IEEEVIS93.ps

Hemmje, Matthias; Kunkel, Clemens; and Willet, Alexander (1994). Lyberworld - a visualization user interface supporting fulltext retrieval. In: Croft,W.B., y
Rijsbergen, C.J. van (eds.) Proceedings of the 17th Annual International Conference on Research and Development in Information Retrieval (SIGIR '94). Dublin, 3-6 July. Springer Verlag, 1994. Pp. 249-257.

ftp://ftp.darmstadt.gmd.de/pub/VISIT/papers/hemmje/SI GIR94.ps

Hemmje, Matthias (1995). Lyberworld - a 3D graphical user interface for fulltext retrieval. Proceedings of $\mathrm{CHI}$ '95.

ftp://ftp.darmstadt.gmd.de/pub/VISIT/papers/hemmje/C HI95.ps.gz

Hendley, R., Drew, N.,Wood, A. and Beale, R. (1995). Narcissus: visualising information. InfoVis '95. Atlanta, IEEE Press. Pp. 90-96.

ftp://ftp.cs.bham.ac.uk/pub/authors/R.J.

Hendley/ieeeviz.ps.Z

Honkela, Timo; Pulkki,Ville; and Kohonen, Teuvo (1995). Contextual relations of words in Grimm tales, analysed by self-organizing map. Proceedings of International Conference on Artificial Neural Networks, ICANN-95. Paris : EC2 et Cie.

http://www.cis.hut.fi/ tho/publications/grimmsom.ps

Honkela, Timo; Kaski, Samuel; Lagus, Krista; and Kohonen, Teuvo. (1996a). Newsgroup exploration with WEBSOM method and browsing interface. Espoo: Helsinki University of Technology, Laboratory of Computer and Information Science. (Technical Report, A32). http://websom.hut.fi/websom/doc/websom.ps.gz

Honkela, Timo; Kaski, Sammuel; Lagus, Krista; and Kohonen, Teuvo (1996b). Self-organizing maps of document collections. Alma. I (2), 1996.

http://www.diemme.it/ /uigi/websom.html

Kaski, Sammuel; and Kohonen, Teuvo (1995).

Exploratory data analysis by the self-organizing map: structures of welfare and poverty in the world. In: Refenes, A. and others (eds.) Neural Networks in Financial Engineering: Proceedings of the Third International Conference on Neural Networks in the Capital Markets. London, II-13 October, 498-507. http://www.cis.hut.fi/ sami/nncm95.ps.gz

Kaski, Sammuel; Honkela, Timo; Lagus, Krista; and Kohonen, Teuvo (1996). Creating an order in digital libraries with self-organizing maps. Proceedings of World Congress on Neural Networks, WCNN '96. Mahwah, NJ: INNS Press, 814-817. http://websom.hut.fi/websom/doc/wenn960.ps.gz 
Kinnucan, Mark; Nelson, Michael; and Allen, Bryce (1987). Statistical methods in information science research. Annual Review of Information Science and Technology (ARIST). 22:147-178, 1987.

Kohonen, Teuvo (1997). Self-organizing maps. 2ed. Berlin : Springer, 1997.

Lagus, Krista; Honkela, Timo; Kaski, Sammuel; and Kohonen, Teuvo (1996). Self-organizing maps of document collections: a new approach to interactive exploration. In: Simoundis, E.; Han, J. and Fayyad, U. (eds.) Proceedings of the Second International Conference on Knowledge Discovery and Data Mining. Menlo Park:

AAAl Press, 238-243.

http://websom.hut.fi/websom/doc/kdd96.ps.gz

Levialdi, Stefano; Massari, Antonio; and Saladini, Lorenzo (1995). Visual metaphors for database exploration. FADIVA 2 Workshop. Glasgow, July 20-22, 1995. http://www-

cui.darmstadt.gmd.de/visit/Activities/Virgilio/Publicatio ns/virgilio.ps

Lin, Xia (1995). Searching and browsing on map displays. Proceedings of ASIS '95. Chicago, October 1995. http://www.uky.edu/ xlin/asis95.htm.

Lin, Xia (1996). Graphical table of contents. Digital Library'96. http://www.uky.edu/ xlin/DL96/DL96.htm.

Lin, Xia (1997). Map displays for information retrieval. Journal of the American Society for Information Science (JASIS). 48(I):40-54, 1997.

Lynch, Patrick (1994).Visual design for the user interface. Journal of Biocommunications. 21 ( 1 ):22-30, 1994.

Moya AnegÛn, FÈlix de (1995). Los sistemas integrados de gestiÛn bibliotecaria: estructuras de datos y recuperaciÛn de informaciÛn. Madrid :ANABAD, 1995. (ColecciÛn Estudios).

Piscitelli, Alejandro (1995). Mente y cuerpo en la era de las m-quinas inteligentes. In: Ciberculturas en la era de las m-quinas inteligentes. Buenos Aires : PaidÛs, 1995. (Contextos).

Salton, Gerard (1979). Mathematics and information retrieval. Journal of Documentation. 35(1): I-29, March 1979.
Library \& Information Research News (LIRN) Volume 22 - Number 71 - Summer 1998

Wood, Andrew; Drew, Nick; Beale, Russell and Hendley, Robert (1995a). Hyperspace: a World-Wide Web visualizer and its implications for collaborative browsing and software agents. Third International WorldWide Web Conference Poster Proceedings. Darmstadt (Germany), 10-14 April 1995. Pp. 21-25. http://www.cs.bham.ac.uk/ amw/hyperspace/www95/

Wood, Andrew; Drew, Nick; Beale, Russell and Hendley, Robert (1995b). Hyperspace: web browsing with visualization. ftp://ftp.cs.bham.ac.uk/pub/authors/R.J.Hendley/1995b. ps.Z 